\title{
Brain-region specific responses of astrocytes to an in vitro injury and neurotrophins
}

\author{
Andrea Beatriz Cragnolini ${ }^{\mathrm{a}}$, Gonzalo Montenegro ${ }^{\mathrm{a}, 1}$, Wilma J. Friedman ${ }^{\mathrm{b}}$, Daniel Hugo Mascó ${ }^{\mathrm{a}, *}$ \\ a IIByT-UNC CONICET, CEBICEM, Facultad de Ciencias Exactas Físicas y Naturales, Universidad Nacional de Córdoba, Av. Vélez Sarsfield 1611, X5016GCA Córdoba, \\ Argentina \\ ${ }^{\mathrm{b}}$ Department of Biological Sciences, Rutgers University, 225 University Avenue, Newark, N.J. 07102, United States
}

\section{A R T I C L E I N F O}

\section{Keywords:}

Astrocytes

BDNF

NGF

Migration

Scratch injury

\begin{abstract}
A B S T R A C T
Astrocytes are a heterogeneous population of glial cells that react to brain insults through a process referred to as astrogliosis. Reactive astrocytes are characterized by an increase in proliferation, size, migration to the injured zone and release of a plethora of chemical mediators such as NGF and BDNF. The aim of this study was to determine whether there are brain region-associated responses of astrocytes to an injury and to the neurotrophins NGF and BDNF. We used the scratch injury model to study the closure of a wound inflicted on a monolayer of astrocytes obtained from cortex, hippocampus or striatum. Our results indicate that the response of astrocytes to a mechanical lesion differ according to brain regions. Astrocytes from the striatum proliferate and repopulate the injury site more rapidly than astrocytes from cortex or hippocampus. We found that the scratch injury induced the upregulation of neurotrophin receptor p75NTR and TrkB.t in astrocytes from all brain regions studied. When astrocytes from all regions were treated with NGF, the neurotrophin induced migration of the astrocytes (assessed in Boyden chambers) and induced wound closure but did not affect proliferation. In contrast, BDNF induced wound closure but only in astrocytes from striatum. Our overall findings show the heterogeneity in astrocyte functions based on their brain region of origin, and how this functional diversity may determine their responses to an injury and to neurotrophins.
\end{abstract}

\section{Introduction}

Astrocytes comprise a population of glial cells that play critical roles during development as well as in the maintenance of the central nervous system (CNS) functions. These glial cells are often defined as a population that shares common characteristics and functions throughout the CNS, however the existence of morphologically different populations of astrocytes has recently been recognized (Schitine et al., 2015). Different astrocytic morphologies are seen not only in resting astrocytes among brain regions but also in response to a lesion, however, the functional consequences of such diversity have not been fully defined. The diversity of astrocytes is also reflected in the factors they release and how they affect their environment. Thus, it has been reported that astroglial cultures obtained from cortex, cerebellum and spinal cord release unique combinations and amounts of chemokines and cytokines (Fitting et al., 2010). The heterogeneity of astrocytes also impacts their synaptogenic potential, mainly due to distinct gene expression profiles (Buosi et al., 2017). Astrocytes undergo morphological and physiological changes during an animal's life (Schitine et al., 2015). Aging and environmental impoverishment significantly affect astrocytes by reducing their morphological complexity (Diniz et al., 2016). Similar changes are observed after different types of injuries. This phenomenon it is called reactive astrogliosis and it is not a homogeneous response. On the contrary, it is highly regulated by contextspecific factors released by neurons, other types of glial cells and astrocytes themselves (Pekny and Pekna, 2014). A CNS injury triggers a finely tuned spectrum of changes ranging from reversible alterations in gene expression and cellular hypertrophy to cell proliferation with compact scar formation and permanent tissue rearrangement (Sofroniew, 2015) and in many cases migration toward the injured site (Zhan et al., 2017).

Reactive astrocytes release a plethora of chemical mediators, including cytokines (Choi et al., 2014), adhesion molecules (Hayakawa et al., 2014), neurotrophins (Patel and Gray, 1993; Rubio, 1997), and extracelular matrix proteins (Wiese et al., 2012) among others. After an injury, neurotrophins can be released by several type of cells, including

\footnotetext{
* Corresponding author.

E-mail address: dmasco@fcq.unc.edu.ar (D.H. Mascó).

${ }^{1}$ Present address: Instituto Multidisciplinario de Biologia Vegetal (IMBIV), Facultad de Ciencias Exactas, Físicas y Naturales, Universidad Nacional de Córdoba, Av. Vélez Sarsfield 299, $2^{\circ}$ piso. 5000 Córdoba
} 
microglia (Gomes et al., 2013; Tay et al., 2016; VonDran et al., 2014) and astrocytes (Goss et al., 1998; Volosin et al., 2006; Domeniconi et al., 2007; Fulmer et al., 2014) as the main sources. Neurotrophins maintain the survival and differentiation of specific populations of neurons in the central and peripheral nervous system and these actions depend on the interaction with specific Trk receptors and/or p75NTR (Park and Poo, 2012). While the effects of neurotrophins on neurons have been extensively studied, little is known about their functions in astrocytes. Neurotrophin receptors have been detected in astrocytes from several brain areas during development (Hutton et al., 1992; Rudge et al., 1994; Cragnolini et al., 2009, 2012), while in the adult brain they are present in a small population of cells and mainly in injured areas (Cragnolini et al., 2009, 2012; Lu et al., 2013; Schachtrup et al., 2015).

There is evidence showing that astrocytes are a target for neurotrophin actions. NGF can modulate the reactivity of astrocytes by reverting the phenotypic changes associated with astrogliosis after a spinal cord injury (Cirillo et al., 2010; De Luca et al., 2016) and can arrest astrocytes in the cell cycle acting through p75NTR (Cragnolini et al., 2012, 2009). On the other hand, BDNF and the truncated TrkB receptor (TrkB.t) modulate the astrocytic morphology in adult rat neocortex (Ohira et al., 2007).

Astrocytic migration is a crucial process in the developing brain for structural organization as well as during pathological conditions (Cayre et al., 2009; Zhan et al., 2017). The scar formation that occurs after an injury consists of both glial (mainly astrocytic) and fibrotic components. It has been shown that the scar formation has both positive and negative consequences for the surrounding nervous tissue. Astrocytes become dynamic migratory cells when they are performing pathological scar formation (Kawano et al., 2012; Zhan et al., 2017) and this response is a highly regulated process that is not fully understood.

In this study we used an in vitro model to compare the closure of a wound inflicted on monolayers of astrocytes obtained from cortex, hippocampus and striatum. We also examined the effects of the neurotrophins NGF and BDNF on the wound closure. Our results indicate that there are region-specific differences in astrocyte responses to an injury and neurotrophins.

\section{Methods}

\subsection{Astrocyte culture}

Astrocytes were obtained from P0-P2 Wistar rats maintained under standard 12-h light-dark cycle, $21{ }^{\circ} \mathrm{C}$ and ad libitum access to food and water. All procedures involving animals were performed in accordance with the international guidelines for the use of laboratory animals, and were approved by the Institutional Committee for Handling and Care of Laboratory Animals. Primary cortical, striatal and hippocampal cultures of astrocytes were performed according to the protocol described by McCarthy and De Vellis (1980). Briefly, cortices, striatum and hippocampi were dissected out from each brain, dissociated by trituration, homogenized and plated on poly-D-lysine-coated $25 \mathrm{~cm}^{2}$ flasks in growth medium: DMEM (Invitrogen, 10\% foetal bovine serum (FBS, Sigma), $50 \mathrm{IU} / \mathrm{mL}$ penicillin and $50 \mathrm{IU} / \mathrm{mL}$ streptomycin (Invitrogen). Astrocytes were grown to confluence and purified by shaking according to previously published methods (McCarthy and De Vellis, 1980; Cragnolini et al., 2012). The astrocytes were trypsinized and replated onto poly-D-lysine-coated coverslips and placed in 24 multiwell plates, $35 \mathrm{~mm}$ plastic Petri dishes or transwell inserts (Corning). The purity of astrocytes was approximately $95 \%$, confirmed by labelling with antibodies against GFAP and devoid of microglia markers staining (antiCD11) and oligodendrocytes progenitors (anti-NG2). To confirm the specificity and functionality of anti-CD11b and anti-NG2 we tested these antibodies on pure microglial cultures and mixed glial cells cultures, respectively (Supplementary Fig. 1). Mixed glial cultures were obtained from primary cultures without the shaking process used to obtain pure astrocytic cultures.

\subsection{Scratch injury}

Astrocytes were plated on glass coverslips $(50,000$ cells per coverslip) and allowed to reach confluence, usually, 7-8 days post-plating.

The day before performing the scratch, the growth medium was aspirated, cells were washed twice with PBS and medium containing low serum was added (DMEM, $1 \% \mathrm{FBS}, 50 \mathrm{UI} / \mathrm{mL}$ penicillin and $50 \mathrm{U} /$ $\mathrm{mL}$ streptomycin). We used low serum concentration to minimize the effect of growth factors in the medium that could mask the effects of the treatments. At the same time, $1 \%$ of FBS allowed the migration induced by the scratch otherwise, in serum free conditions, migration occurs at very low speed even in the presence of neurotrophins.

Twenty four hours later one longitudinal scratch was made in each well using a sterile plastic pipette tip (Etienne-Manneville, 2008), The multiwell plates containing astrocytes were washed once with PBS to remove the debris and incubated with fresh media with low serum and containing the treatments. Immediately following the addition of treatments (time 0), pictures were taken using an inverted microscope in bright field (Nikon Te2000-U). Astrocytes were maintained for $48 \mathrm{~h}$ after the scratch and pictures were taken at the end of the period to calculate the area covered by cells post-injury. Ten to twelve pictures were taken along scratch with a $10 \times$ magnification objective and superimposing approximately $10 \%$ between each contiguous image. Individual images were stitched to reconstruct the scratch at time $0 \mathrm{~h}$ and $48 \mathrm{~h}$ and the cell-free area was calculated using the FIJI software (Schindelin et al., 2012) to estimate the wound closure.

\subsection{SDS-PAGE and western blot}

For western blot analysis for neurotrophin receptors expression, confluent astrocyte monolayer grown in $35 \mathrm{~mm}$ dishes, were scratched with pipette tips 6 times in one direction followed by 6 times at 90 degree angles to the first set of scratches to increase the area exposed to injury. Twenty four hours later astrocytes were lysed in RIPA buffer, proteins quantified and Western blot was run in standard conditions.

To assess the expression of NGF and BDNF whole cell lysate of astrocytes were used. Cells were lysed in RIPA buffer, proteins quantified and were run in SDS-PAGE, proteins were transferred to a nitrocellulose membrane. The membranes were fixed with $2.5 \%$ glutaraldehyde for $30 \mathrm{~min}$, this step was required to detect low molecular weight proteins (Anastasia et al., 2013) and we did not detect NGF or BDNF without the fixation.

\subsection{Assessment of astrocyte viability post-scratch injury}

To assess cell viability post-scratch injury in astrocyte cultures from cortex, hippocampus and striatum were stained with calcein AM/ethidium (Molecular Probes, Cat \# mp03224) according to manufacturer's instructions. Briefly, cells from each brain area were cultured on coverslips and scratched as described above, then astrocytes were washed twice with PBS and a mix of calcein AM/ethidium was added and incubated for $25 \mathrm{~min}$ at room temperature. Immediately after coverslips were mounted with FluorSave on glass slides and observed under a fluorescence microscope. Five photos per coverslip were taken along the scratch covering a distance of 650 um from de edge of the scratch. Astrocyte death rate was calculated by determining the percentage of ethidium-positive cells over total cell number. For each experimental group, five fields from at least two coverslips from two separated cultures were imaged were evaluated for each brain region.

\subsection{Proliferation assay}

To analyze the proliferation rate of astrocytes along the edge of the injury, astrocytes were grown to confluence and scratched as described 
above. BdrU $(10 \mu \mathrm{M})$ was added to the cultures together with the treatments. Forty-eight hours later, cells were fixed with $4 \%$ paraformaldehyde and immunostained using a specific antibody against BrdU as described in Cragnolini et al. (2009). Pictures were obtained from fields of equivalent size in three different areas of scratched monolayers.

\subsection{Migration assay}

The experiments were conducted using P0-P2 Sprague-Dawley rats p75NTR homozygous knockout rats $(p 75 N T R-/-)$ generated by CRISPR technology (Sage Labs/Horizon, USA) or wild type ( $p 75 N T R$ $+/+$ ). The p75NTR $-/-$ rats were genotyped by PCR to confirm the lack of p75NTR. Cell migration and chemotactic effect of neurotrophins was assayed using transwell chambers containing an $8 \mu \mathrm{m}$ pore size filter (Corning, cat \#3464), following the supplier's directions. Briefly, $15 \times 10^{4}$ cells were plated on the poly-D-lysine-coated upper chamber. $24 \mathrm{~h}$ later cells were washed with PBS and medium replaced with medium with low serum, and neurotrophins were added to the lower chamber and maintained for $24 \mathrm{~h}$. At the end of the period, filters were removed from the incubator and the remaining cells on the upper chamber were detached with a cotton swab. The cells on the lower side of the filters were fixed with $4 \%$ paraformaldehyde and stained with DAPI. The filters were then placed on glass slides ensuring that the lower surface of the filter was adjacent to the glass slide. One picture per filter was taken with a $4 \times$ magnification objective to cover most of the filter surface. The number of cells which migrated to the lower side of the filter were automatically counted with FIJI software (Schindelin et al., 2012).

\subsection{Adhesion assay}

We performed the adhesion assay as described by Giralt et al. (2016). Briefly, confluent cultured astroglia were trypsinized and suspended in DMEM with 10\% FBS. After measurement of cell density with a hemocytometer, $5 \times 10^{4}$ cells per well were plated in 24-well plates coated with poly-D-lysine. Four hours after plating cells were washed with PBS to remove non-adherent cells, lysed and intact cell nuclei were counted with a hemocytometer, a quantification method we have used previously (Friedman, 2000; Volosin et al., 2006). Nuclei of dead or dying cells are irregularly shaped or disintegrated. In contrast, nuclei of healthy cells are phase bright and have clearly defined limiting membranes. Cell counts were performed in triplicate and adhesion rate was defined as the percentage of cells remaining $4 \mathrm{~h}$ after plating.

\subsection{Chemicals}

Recombinant human NGF (hNGF) and hBDNF were purchased from Alomone (NGF, cat \# N-245; BDNF, cat \# B-250). Culture media was from Invitrogen, poly-D-lysine, ANA-12 and FBS were purchased from Sigma. Antibody against BrdU was from Millipore (Cat\# MAB3510 RRID:AB_94897, dilution 1:50), anti-GFAP from Immunostar (Cat\# 22522 RRID:AB_572240, dilution 1:8), anti-CD11 (Cat\# CBL1512Z RRID:AB_347694, dilution 1:100), anti-NG2 (Cat\# sc-33,666, dilution 1:50) and anti-p75NTR for blocking assay (Cat\# MAB365 RRID:AB_ 2152788, dilution 1:500) were from Millipore, anti-p75NTR Rex used for Western blot was kindly provided by Dr. Louis Reichardt (dilution 1:2000) anti-TrkB was from Santa Cruz Biotechnology (Cat\# sc-8316 RRID:AB_2155274, dilution 1:1000) anti-BDNF (Cat\# sc-546 RRIB:AB_630940, dilution 1:500) and anti-NGF (Cat\# sc-548 AB_632011, dilution 1:500).

\subsection{Statistics}

The results are expressed as mean \pm standard deviation (SD), unless otherwise is indicated, of at least 3 independent cell cultures.
ANOVA was used to compare groups and was followed by Tuckey post hoc comparisons, with $p \leq 0.05$ considered significant.

\section{Results}

\subsection{Scratch injury induces a brain region-dependent astrocytic response}

In order to begin to understand the role of neurotrophins and their receptors in the wound closure by astrocytes, we first compared how astrocytes from cortex, striatum and hippocampus responded to the scratch injury inflicted to a cell monolayer. The injury left an empty area of 550-600 $\mu \mathrm{m}$ which was then closed by growing astrocytes. We found that there was a regional heterogeneity in the astrocyte response to the wound. Astrocytes from the three studied areas showed different speed of closure of the injured area (Fig. 1a, b). Thus, striatal astrocytes were the fastest cells to progress toward the injured site, occupying $63 \pm 4,3 \%$ of the initial empty gap at $48 \mathrm{~h}$ post-scratch. In contrast, in cultures from hippocampus and cortex the occupied areas were significantly lower than striatum, $54,5 \pm 6,1 \%$ and $39,43 \pm 5,6 \%$, respectively. Since the cultures were highly pure populations of astrocytes, confirmed by immunostaining of GFAP, we rule out the possibility that other glial cell types can contribute to the closure of the wounded astrocytic cultures obtained from the three brain areas (Supplementary Fig. 1).

\subsection{Striatal astrocytes show higher proliferation rates than cortex and hippocampus in response to an injury}

The repair process involves both cell proliferation along the edge of the injury and migration of astrocytes toward the injured site. We investigated whether the differences in wound closure were inherent to the astrocytes from the different brain regions, and whether they were due to differences in proliferation or migration. Cultures of astroglial monolayers obtained from cortex, hippocampus or striatum were scratched and incubated with Brdu for 12, 24, 48 and $72 \mathrm{~h}$ following injury. At the end of each incubation period, the cultures were subjected to immunostaining and the proportion of the BrdU-positive cells along the scratch and occupying the cell-free gap was calculated as a percentage of the total nuclei in a given microscopic field. In agreement with previous publications (Környei et al., 2000; Yang et al., 2009; Cragnolini et al., 2012) we observed that the injury caused a significant increase in the proportion of proliferating cells along the wound which decreased with the distance to the cell-free gap (Fig. 2a, c). The population in the center of the injury and the invading cells into the gap at $48 \mathrm{~h}$ was mainly dominated by proliferating astrocytes obtained from striatum (92\%) and cortex (50\%), but the proliferating cells in the gap of hippocampal cells was only 17\% (Fig. 2c).

We also found that the proportion of proliferating cells over the time along the scratch varied among the astrocytes from different brain areas. Cell growth curve demonstrated that astrocytes obtained from striatum have a higher proliferation rate compared to astrocytes derived from cortex and hippocampus. This difference started to be significant $24 \mathrm{~h}$ post-injury and it was sustained over the evaluated period. Astrocytes from cortex showed a slightly higher proliferative rate respect to astrocytes derived from hippocampus that was significant at $72 \mathrm{~h}$ post-scratch.

To assess whether astrocytes from different brain region have a different susceptibility to death following the mechanical injury we carried out a survival/death assay staining with calcein-AM and ethidium homodimer-1. Viable cells exhibited green fluorescence generated by the esterase hydrolysis of membrane-permeant dye, calcein-AM and dead cells were marked by red fluorescence ethidium homodimer-1 (Fig. 2d, e). The percentages of dead cell along the edge of the scratch were $2.97 \pm 0.22 ; 2.83 \pm 0.53$ and $2.76 \pm 0.19$ for astrocytes from cortex, hippocampus and striatum, respectively. There were no significant differences on cell death between injured astrocytes obtained 
a

Oh

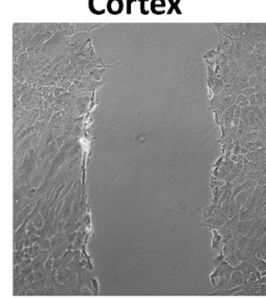

$48 \mathrm{~h}$

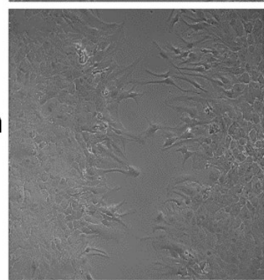

Hippocampus

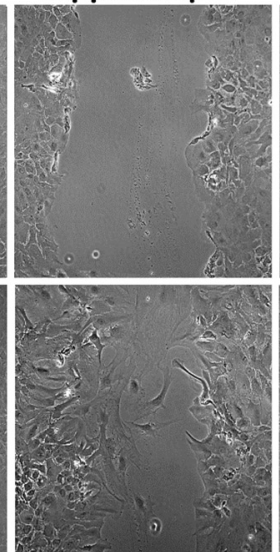

\section{Striatum}

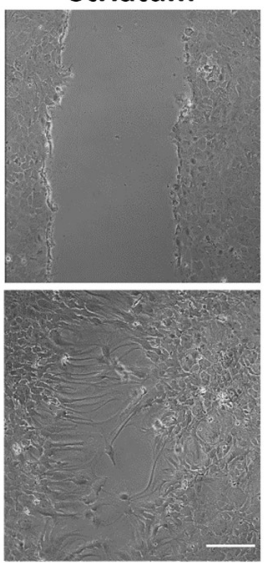

b

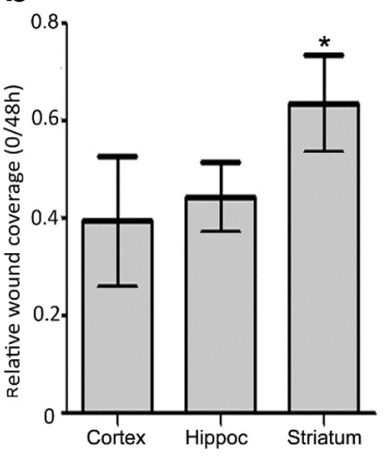

Fig. 1. Different wound occupation in astrocytic cultures from distinct brain areas. a) Representative images of wound healing in monolayers of astrocytes from cortex, hippocampus and striatum, upper row shows images of astrocytes immediately after the injury (time 0 ), bottom row, $48 \mathrm{~h}$ after the injury. b) Quantification of the occupied area $48 \mathrm{~h}$ after the injury. Mean $\pm 95 \%$ confidence interval, $n \geq 5, * p \leq 0.05$. Scale bar $200 \mu \mathrm{m}$ from the three different areas.

\subsection{Neurotrophin receptors $p 75 N T R$ and TrkB.t are up-regulated in} astrocytes from cortex, hippocampus and striatum after a scratch injury

We previously demonstrated that in vivo brain injuries, such as status epilepticus, or an in vitro scratch wound induced the up-regulation of
p75NTR expression in astrocytes from hippocampus (Cragnolini et al., 2009, 2012). It has also been shown that TrkB.t1, the main isoform of the TrkB receptor expressed in astrocytes, is up-regulated in these cells after a spinal cord lesion (Matyas et al., 2017). To explore whether the increase in the expression of both receptors, p75NTR and TrkB.t, is a common hallmark of astrocytes from different brain areas after a mechanical injury, we performed a scratch wound on astrocytic a
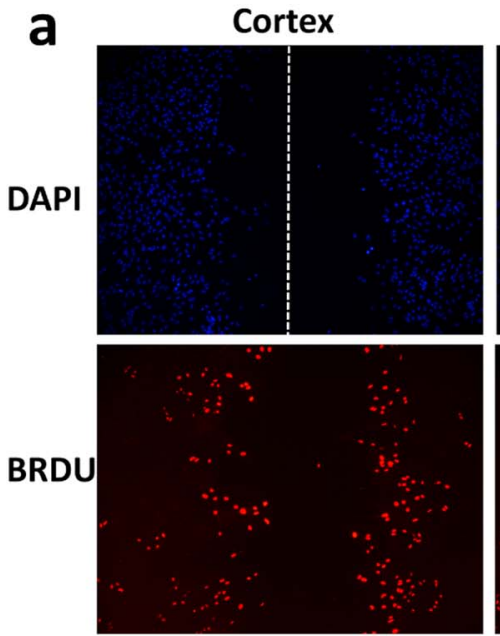

b

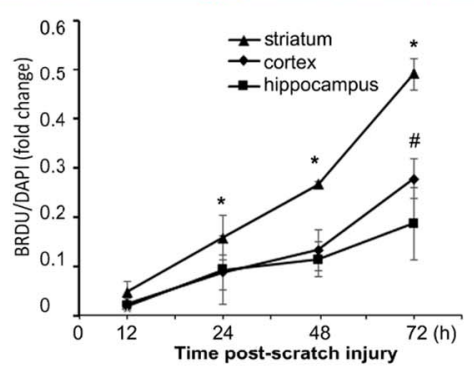

d

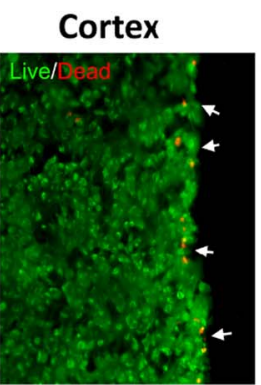

Hippocampus

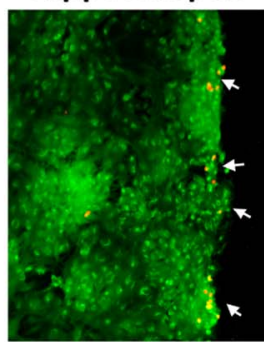

Hippocampus
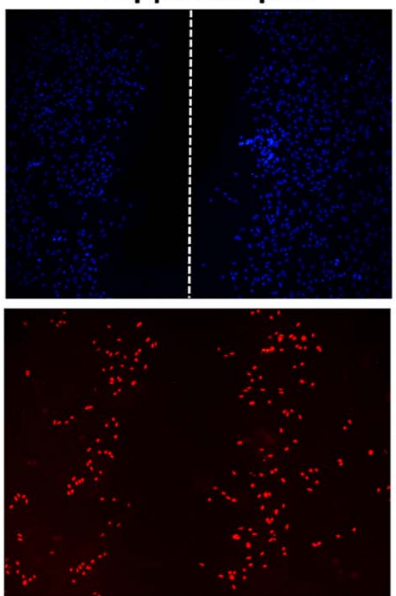

C

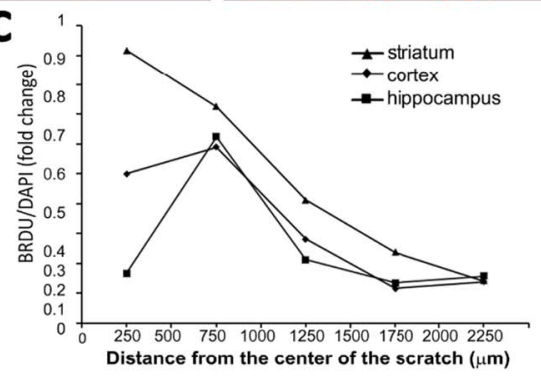

Striatum
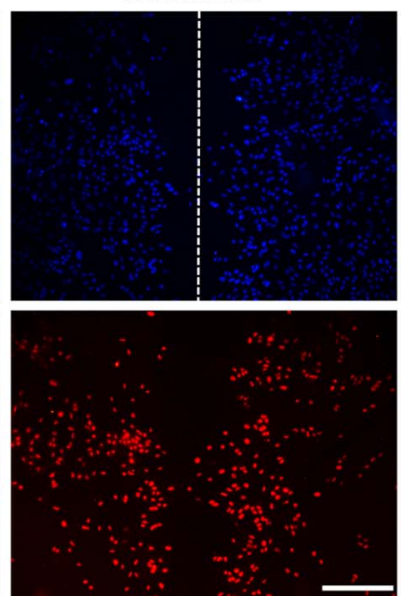

Striatum

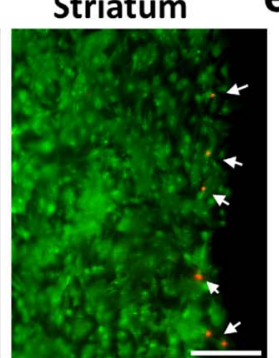

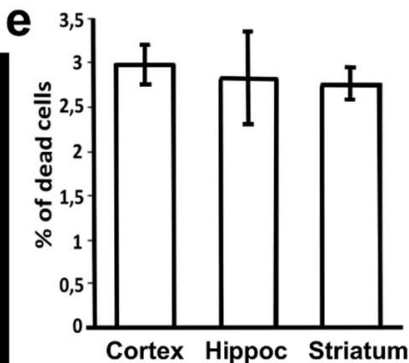

Fig. 2. Scratch wound induces proliferation of astrocytes in a brain region-dependent manner. Astrocytes obtained from cortex, hippocampus or striatum were injured and immediately provided with $\operatorname{BrdU}(10 \mu \mathrm{M})$ for $48 \mathrm{~h}$, then fixed and labeled for BrdU and DAPI. a) Photomicrographs of dual staining for DAPI (top row) and BrdU (bottom row) along the scratch-wounded astrocytes from cortex, hippocampus and striatum. b) Quantification of astrocyte proliferation ratio in wounded regions at $12,24,48$ and $72 \mathrm{~h}$ post-injury. Mean \pm SEM, $n=3,{ }^{*} p \leq 0.05$ striatum $v s$ hippocampus or cortex, \# $p \leq 0.05$ cortex $v s$ hippocampus. c) Lateral distribution of proliferating cells in wounded areas; consecutive images were taken along $2250 \mu \mathrm{m}$ from the center of the scratch (dotted line in Fig. 2a) and the ratio Brdu/DAPI was quantified. d) Representative micrographs showing astrocytic cultures stained with calcein/ethidium immediately after the scratch injury (calcein + cells, green; ethidium homodimer-1 + cells, red, are indicated with white arrows). e) Quantification of dead cells along the edge of the wound. Mean \pm SEM, $n=2$. Scale bar $200 \mu \mathrm{m}$. (For interpretation of the references to colour in this figure legend, the reader is referred to the web version of this article.) 

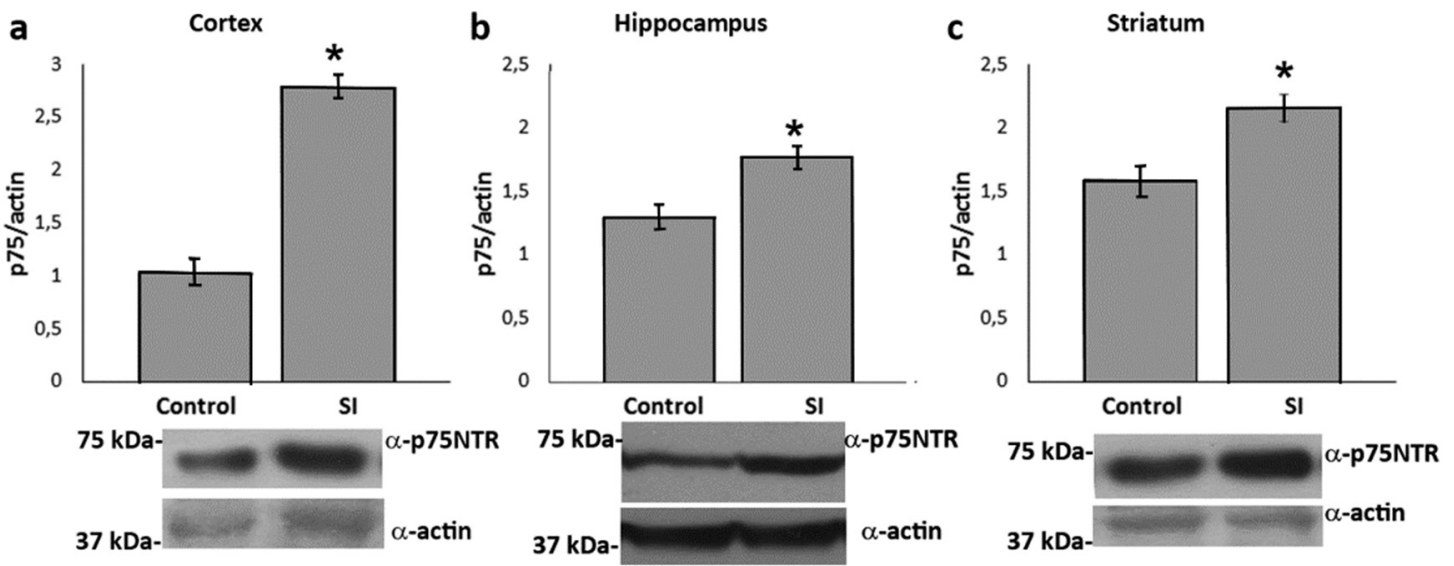

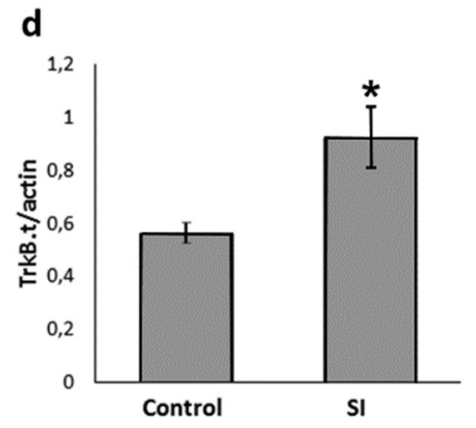

e
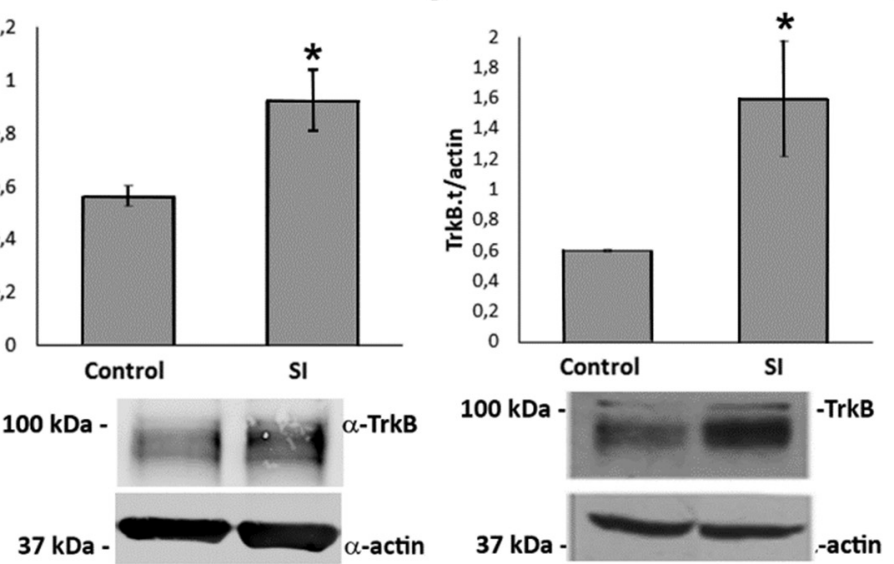

f

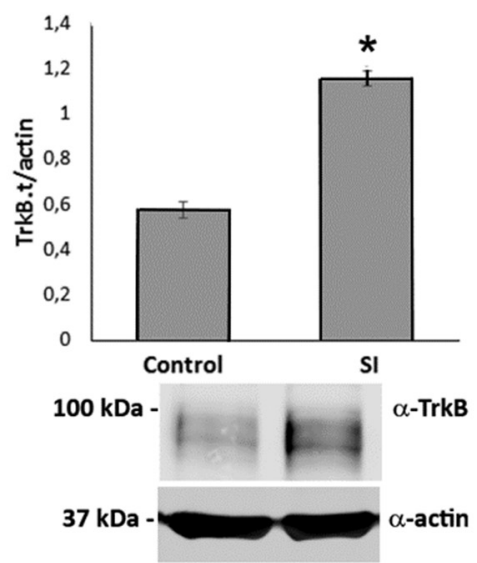

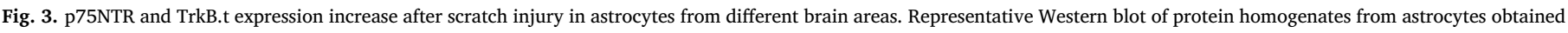

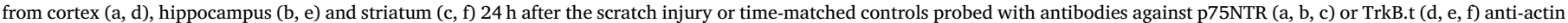
was used as a loading control. Top panels, densitometric quantification of Western blot bands expressed as ratio of p75NTR or TrkB/actin. Mean \pm S.E.M., $n=3$, * $p \leq 0.05$.

monolayers and $24 \mathrm{~h}$ later the levels of these receptors were analyzed. Western blot analysis demonstrated that non-injured astrocytes obtained from cortex, hippocampus and striatum express p75NTR and TrkB.T1 receptors (Fig. 3). The in vitro injury caused a significant upregulation in the expression of both receptors, p75NTR and TrkB.t on astrocytes from the three brain areas studied. To detect TrkB on astrocytes by Western blot we used an antibody that recognizes the full length and the truncated isoforms (Cat\# sc-8316 RRID:AB_2155274) and we only detected two bands at approximately $90 \mathrm{kDa}$ corresponding to the truncated isoforms on astrocytes from the three brain regions.

\subsection{Exogenous neurotrophins induce wound closure of astrocytic monolayers without affecting proliferation}

We next examined the effect of NGF and BDNF on wound closure. Astrocytes were scratched and NGF or BDNF $(10 \mathrm{ng} / \mathrm{mL})$ were added immediately afterward. Treatment with NGF increased the speed of invading astrocytes obtained from the three brain areas studied (Fig. 4) compared to untreated controls. The effect of NGF was more noticeable in cortical astrocytes (Fig. 4a) which increased the occupied cell-free area $48 \%$ more than controls. In astrocytes from hippocampus and striatum the occupied area post-scratch after treatment with NGF were, respectively, $25 \%$ and $20 \%$ larger than controls. We also assessed the effect of BDNF (10 ng/mL) on wound closure but unlike NGF, only induced an increase in the occupied area post-injury in astrocytes from striatum.

Astrocytes from different brain areas progressed at different rate toward the gap, however all of them had the capability to close the wound. When we observed astrocytes at $72 \mathrm{~h}$ porst-sctach (Supplementary Fig. 2) the gaps were completely closed in cell cultures treated with NGF in cortical and hippocampal astrocytes and in the striatum were tightly closed with neurotrophins (Supplementary Fig. 2) that was difficult to distinguish the astrocytes that invaded the scratch from the nearby non-injured cells.

To determine whether the endogenous production of neurotrophins NGF and BDNF varies among astrocytes from different brain regions we analyzed their expression by Western blot in whole cell lysate of uninjured astrocytes. To detect the neurotrophins we used a protocol that includes a fixation of the proteins transferred to the nitrocellulose membranes with glutaraldehyde (Anastasia et al., 2013) otherwise we did not observe any band in our samples (Supplementary Fig. 3). We observed dim bands at approximately $14 \mathrm{kDa}$ corresponding to BDNF as well as a higher molecular weight bands that could correspond to proNGF. When we probed our blots with anti NGF we did not detect mature NGF, however we detected a single band between 20 and $25 \mathrm{kDa}$. We did not observe differences in the levels of expression of NGF or BDNF between astrocytes from cortex and striatum.

The results obtained so far indicate that astrocytes from the cortex and hippocampus responded similarly to the scratch injury as well as to the treatment with neurotrophins. For that reason we decided to continue with the studies comparing only astrocytes of cortex and striatum.

The wound closure mainly depends on cell migration and proliferation. It was therefore important to determine which of these processes was affected by neurotrophins. We analyzed the proliferation of wounded astrocytic monolayers treated with NGF or BDNF by 

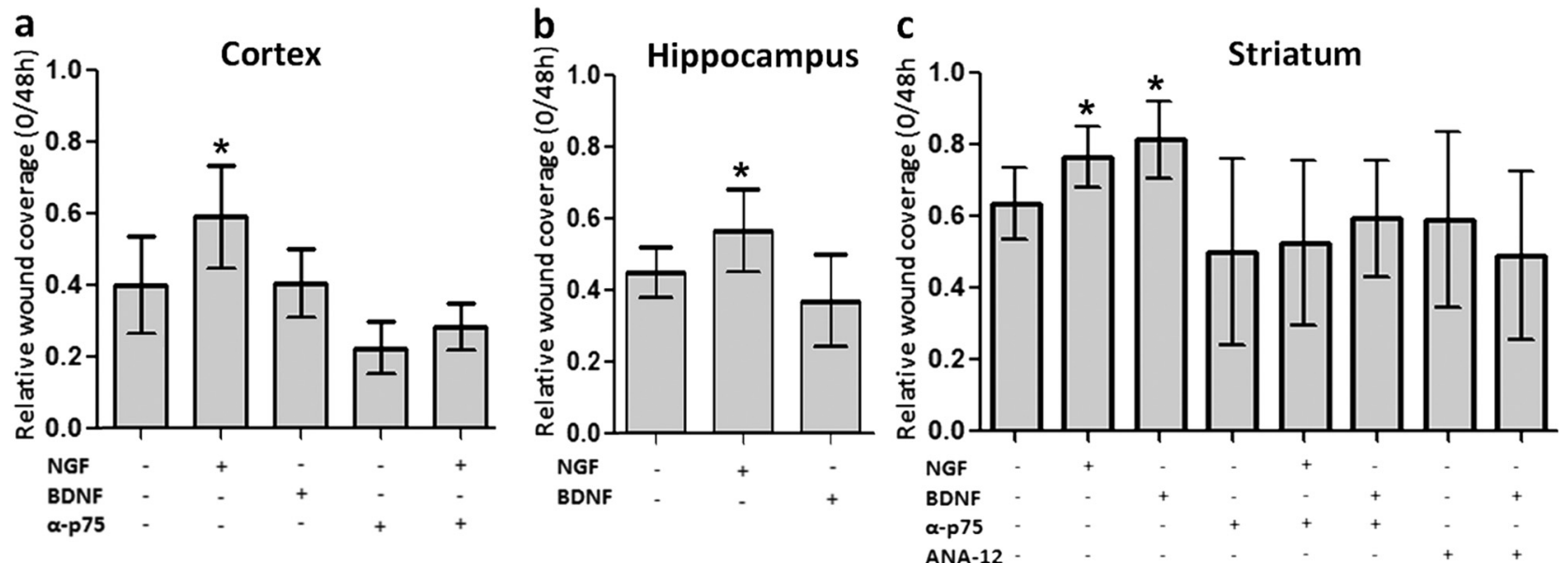

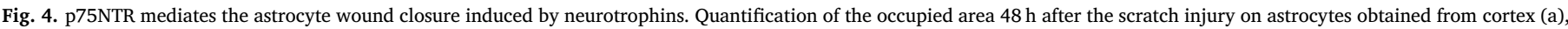

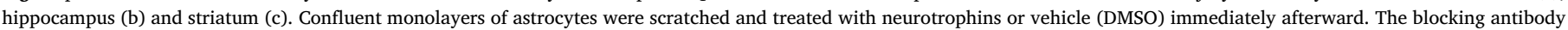

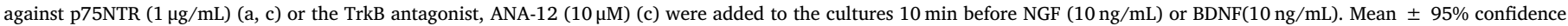
interval, $n \geq 5 * p \leq 0.05$.

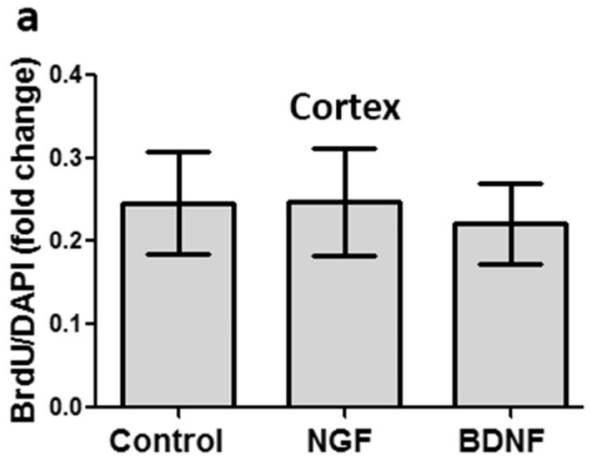

b

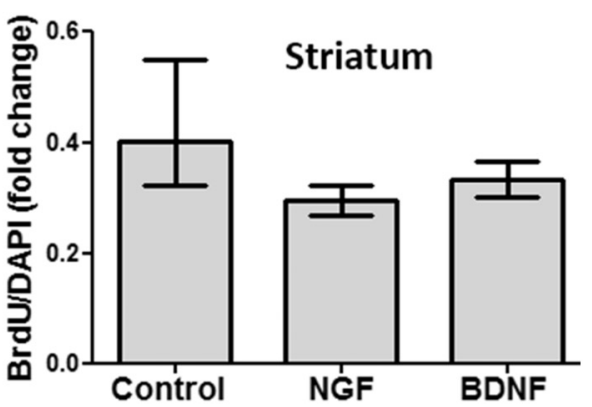

Fig. 5. Neurotrophins did not affect the scratch injury-induced proliferation in astrocytes from cortex and striatum. Astrocytes obtained from cortex (a) or striatum (b) were injured and treated with NGF or BDNF immediately afterward $(10 \mathrm{ng} / \mathrm{mL})$ and provided with BrdU $(10 \mu \mathrm{M})$ for $48 \mathrm{~h}$. Astrocytes were fixed and labeled for BrdU and DAPI and the proliferation rate was quantified as the ratio of BrdU positive cells to DAPI. Mean $\pm 95 \%$ confidence interval, $n=3$, ${ }^{*} p \leq 0.05$. quantifying the incorporation of BrdU along the edge of the injured area. We observed that none of the neurotrophins affected the proliferation rate induced by the scratch injury in astrocytes from cortex (Fig. 5a) or striatum (Fig. 5b).

\subsection{P75NTR is required for NGF and BDNF-induced astrocytic wound closure}

To determine whether the p75NTR receptor mediated the effects of NGF or BDNF on wound closure of astrocytic monolayers we added a function-blocking antibody against p75NTR $10 \mathrm{~min}$ before the neurotrophins and the occupied areas were measured at $t=0$ and $48 \mathrm{~h}$ posttreatment. Exposure of the astrocytes to anti-p75NTR $(0.5 \mu \mathrm{g} / \mathrm{mL})$ followed by NGF or BDNF $(10 \mathrm{ng} / \mathrm{mL})$ reversed the effects on wound closure of NGF on astrocytes from cortex and striatum, and BDNF on astrocytes from striatum (Fig. 4a,c). Similarly, to assess whether TrkB.t was involved in the effect of BDNF on wound closure of astrocytes from striatum we used the specific TrkB antagonist ANA-12 (10 $\mu \mathrm{M})$ (Cazorla et al., 2011) administered $10 \mathrm{~min}$ before BDNF. The addition of ANA-12 immediately after the scratch abolished the effect of BDNF on wound closure of striatal astrocytes (Fig. 4c).

\subsection{The NGF-induced migration is abolished in p75NTR - / - astrocytes}

We showed that NGF induced the wound closure of injured astrocytes without affecting the proliferation (Figs. 4 and 5). Although several methods are used to study migration, the Boyden chamber assay is one of the most accepted methods, therefore we utilized this assay to confirm our results obtained with the scratch injury. To ascertain the effect of NGF on migration, a chemotaxis and migration assay using transwell chambers was carried out in non-injured cortical and striatal astrocytes obtained from wild type and p75NTR $-/-$ rats. We used NGF $(10 \mathrm{ng} / \mathrm{mL})$ or DMEM with $1 \%$ FBS as the control group in the lower chamber. When NGF was added for $24 \mathrm{~h}$ the number of astrocytes migrating through the $8 \mu \mathrm{m}$ pore filter increased 2.5 times in cortical astrocytes and 1.6 times in striatal astrocytes compared to control. The NGF-induced migration was abolished in p75NTR $-/-$ astrocytes obtained from cortex and striatum (Fig. 6).

To rule out the possibility that the decrease in astrocytic migration in $\mathrm{p} 75^{\mathrm{NTR}} \mathrm{KO}$ were due to an adhesion deficit we performed an adhesion assay in astrocytes from cortex and striatum. We found that wild type and KO astrocytes obtained from both regions displayed similar adhesion to a poly-D-Lysine-covered substrate (data not shown). These results indicate that the migratory deficit of p75NTR $-/-$ astrocytes are not related to adhesion deficits.

\section{Discussion}

In this study we used an in vitro scratch-wound model to demonstrate that astroglia derived from different regions differ in their responses to a mechanical injury and to the neurotrophins, NGF and BDNF. The scratch injury has been extensively employed to study wound healing and migration, since it recapitulates most of the main astrocytic responses to an in vivo injury such as polarization of cell processes, migration and proliferation along the edge of the scratch (Etienne-Manneville, 2008; Cragnolini et al., 2012). In our study, the scratch injury was made on completely confluent astrocytic cultures and maintained for the same time in vitro, suggesting that the 

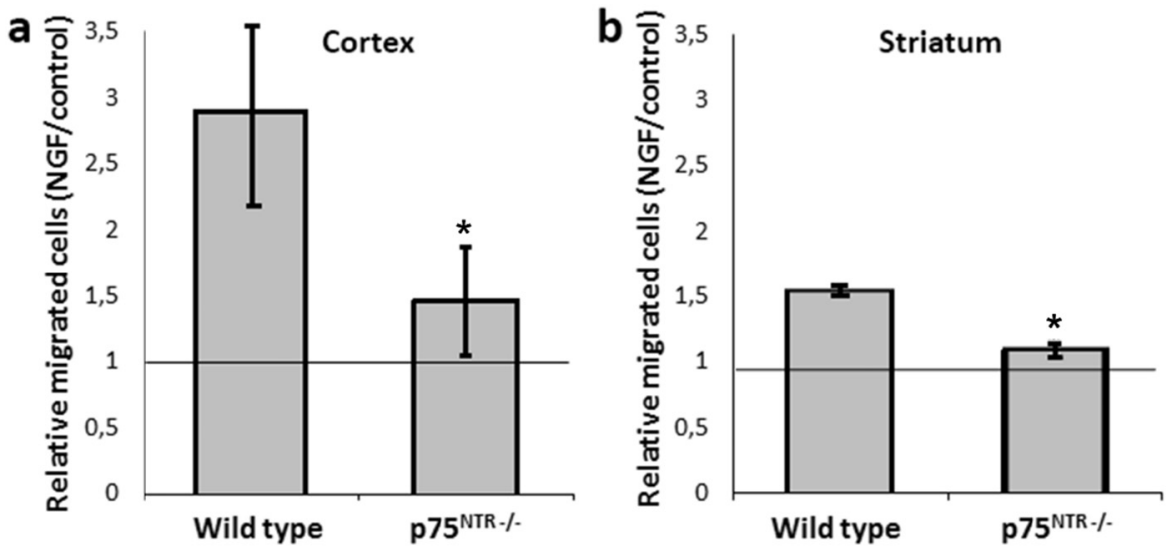

Fig. 6. p75NTR mediates the NGF-induced migration in astrocytes from cortex and striatum. Astrocytes obtained from cortex (a) or striatum (b) were plated on top of a transwell chamber and migration to the bottom side of the filter was quantified $24 \mathrm{~h}$ after the addition of NGF $(10 \mathrm{ng} / \mathrm{mL})$ to the lower chamber. Results are expressed as the ratio of migrating cells treated with NGF respect to controls. Mean \pm SEM, $n=3 * p \leq 0.05$ differences observed correspond to inherent properties of astrocytes from different regions and not to the age of cultures or confluence levels.

Our results demonstrate that astrocytes obtained from cortex, hippocampus and striatum can respond to the scratch injury by proliferating and invading the empty space that it is left by the lesion. This response was not uniform among the astrocytes derived from different brain regions. Thus, astrocytes obtained from striatum occupied the empty gap post-injury faster than astrocytes from the other brain regions. Astrocytes from cortex and hippocampus were also able to close the injury but it took longer than those from the striatum, indicating that there were temporal differences in the reaction to the injury instead of a fail to respond. In addition, the temporal pattern of proliferation post-scratch injury showed a localized response along the edge of the wound where striatum derived astrocytes proliferated more than those obtained from hippocampus or cortex. Other studies have shown that proliferation of astrocytes varies among brain regions in the healthy adult mouse brain, and the hippocampus was one of the areas with the highest mitotic index (Emsley and Macklis, 2006). The variation may be due to methodological issues such as the type of animals used for the study and the fact that they analyzed the proliferation of astrocytes in vivo where the environment is extremely complex and numerous factors may contribute to the response to injury. Together, our results show that the response of astrocytes to a lesion is heterogeneous, and suggest that striatal astrocytes could have a greater capability for wound repair in vitro.

At this point is unclear what determines and maintains the brain region differences of astrocytes, but many environmental cues may be involved in determining the astrocytic phenotypes such as the extracellular matrix (Johnson et al., 2015; Dauth et al., 2016) or the availability of trophic factors (Das et al., 2001). We demonstrated that the scratch injury induced an increase in the levels of the neurotrophic receptors p75NTR and TrkB.t of astrocytes from the three brain areas. This observation suggests that the upregulation of p75NTR and TrkB.t, could be a distinctive response of all astrocytes to different types of CNS injuries and make them more sensitive to neurotrophins. This idea is also supported by our previous studies on the expression of p75NTR in injured hippocampal astrocytes in vitro (Cragnolini et al., 2012) and animals that underwent status epilepticus (Cragnolini et al., 2009). Others have also shown the upregulation of p75NTR in astrocytes after different kind of brain insults (Oderfeld-Nowak et al., 2003; VonDran et al., 2014) as well as TrkB.t1 (Matyas et al., 2017; Wu et al., 2013). Neurotrophins, released by neurons and glial cells, are important signals for migration during development and in the course of CNS repair (Bartkowska et al., 2010; Grade et al., 2013; Oliveira et al., 2013). In this work we did not observe differences in neurotrophin expression among non-injured astrocytes in culture, however it is known that their levels show differential patterns in the rat brain regions during development and adulthood (Das et al., 2001) this could influence on the variability of astrocytic responses in pathological conditions. Further studies would be needed to determine whether the pattern of expression of neurotrophins is affected by an injury in astrocytes from different brain areas.

We demonstrated that neurotrophins added to a confluent glial monolayer has a differential effect on astrocytes from distinct brain areas after a scratch injury. While NGF promoted the repopulation of the wound in astrocytes from all brain areas, BDNF only stimulated the striatal astrocytes. An effect of NGF and BDNF on migration of multipotent astrocytic stem cells has been reported earlier (Douglas-escobar et al., 2012). Although they used higher concentrations of NGF, ranging from 200 to $400 \mathrm{ng} / \mathrm{mL}$ to induce the wound closure. This discrepancy may stem from differences in the cell types used in their studies which could have a different sensitivity to NGF. Both neurotrophins, NGF and BDNF, are known to affect proliferation of several cell types (Cragnolini et al., 2009; Cragnolini et al., 2012; Islam et al., 2009; Tu et al., 2017; Waterhouse et al., 2012). Our results demonstrated that the concentration used to induce wound closure did not affect the proliferation induced by the injury.

Using p75KO rats and Boyden chambers to assess the migration of astrocytes, we observed that the absence of p75NTR markedly alters astrocyte migration induced by NGF. This result is in agreement with data obtained using a blocking antibody in the scratch injury assay. NGF binds to both TrkA and p75NTR receptors, however we have previously shown that TrkA is not expressed in astrocytes (Cragnolini et al., 2009), thus we rule out the possibility that this receptor could be involved in NGF effects. Consistent with our results, p75NTR mediates the effect of NGF and BDNF on migration of several cell types, including Schwann cells (Anton et al., 1994), neuroblasts (Grade et al., 2013) and many tumor cells (Bapat et al., 2016; Shonukan et al., 2003). The results obtained so far rule out the possibility that the induction of wound closure is due to an effect on the proliferation of astrocytes suggesting that neurotrophins affect specifically migration instead of proliferation.

On the other hand, BDNF can bind to both neurotrophin receptors, p75 and TrkB.t. We showed that inhibiting either p75NTR with an antibody or TrkB.t with the antagonist ANA-12 caused the effect of $\mathrm{BDNF}$ to be blocked in astrocytes from striatum. Since we reversed the effect of BDNF on striatal astrocytes by either blocking TrkB.t or p75NTR we suggest that both receptors are necessary for mediating the BDNF in astrocytes from striatum. In agreement with our results, Matyas et al. (2017), recently observed that TrkB.t1 mediated the scratch injury closure induced by BDNF or serum on astrocytes from spinal cord.

It would be interesting to study if both receptors physically interact and stimulate signaling pathways associated with migration in astrocytes from striatum. In fact, previous studies showed that the interaction of p75NTR and TrkB.t is necessary to induce the formation of filopodia in neurons (Hartmann et al., 2004). It is unknown what makes astrocytes from different brain regions sensitive to distinct 
neurotrophins. It is known that p75NTR interacts with other receptors, such as Trk receptors, sortilin and SorCS1, and recruits various cytoplasmic proteins to trigger different signaling pathways (VonDran et al., 2014). It would be interesting to determine whether these co-receptors and interacting proteins have a selective distribution in the CNS that may explain the regional responses of astrocytes to neurotrophins.

The potential consequences of the upregulation of neurotrophic receptors in astrocytes could involve effects on astrocytes itself as well as on the surrounding cells. The upregulation of p75NTR and TrkB.t might increase the migration of astrocytes stimulated by neutrophins facilitating the scar formation, which has recently been demonstrated that supports the axonal growth (Anderson et al., 2016). On the other hand, the activation of TrkB.t by BDNF in astrocytes triggers the release of nitric oxide which has detrimental consequences for neurons (Colombo et al., 2012).

Our overall findings show the heterogeneity in astrocyte functions based on their brain region origin and how this functional diversity may determine their responses to an injury and to neurotrophins. Knowing the mechanisms triggered during wound closure in different brain areas could help to develop new strategies to repair CNS damage. Further studies are clearly needed to determine in vivo whether neurotrophins have a brain-region associated effect, and could help to develop new strategies to repair specific CNS areas.

Supplementary data to this article can be found online at https:// doi.org/10.1016/j.mcn.2018.02.007.

\section{Acknowledgment}

This study was supported by grants from CONICET (11420110100226), PICT [2011 \#1859, 2012 \#2100 and 2015 \#3199] and SECyT-UNC (113/17). GM was a recipient of a fellowship from Consejo Interuniversitario. We thank to CONICET for a travel grant given to $\mathrm{ABC}$ to perform some of the experiments at the Department of Biological Sciences, Rutgers University, Newark, NJ, USA. The authors declare no competing financial interest.

\section{References}

Anastasia, A., Deinhardt, K., Chao, M.V., Will, N.E., Irmady, K., Lee, F.S., Hempstead, B.L., Bracken, C., 2013. Val66Met polymorphism of BDNF alters prodomain structure to induce neuronal growth cone retraction. Nat. Commun. 4, 1-12. http://dx.doi.org/ 10.1038/ncomms3490.

Anderson, M.A., Burda, J.E., Ren, Y., Ao, Y., O'Shea, T.M., Kawaguchi, R., Coppola, G., Khakh, B.S., Deming, T.J., Sofroniew, M.V., 2016. Astrocyte scar formation aids central nervous system axon regeneration. Nature 1-20. http://dx.doi.org/10.1038/ nature17623.

Anton, E.S., Weskamp, G., Reichardt, L.F., Matthew, W.D., 1994. Nerve growth factor and its low-affinity receptor promote Schwann cell migration. Proc. Natl. Acad. Sci. U. S. A. 91, 2795-2799. http://dx.doi.org/10.1073/pnas.91.7.2795.

Bapat, A.A., Munoz, R.M., Von Hoff, D.D., Han, H., 2016. Blocking nerve growth factor signaling reduces the neural invasion potential of pancreatic cancer cells. PLoS One 11, e0165586. http://dx.doi.org/10.1371/journal.pone.0165586.

Bartkowska, K., Turlejski, K., Djavadian, R.L., 2010. Neurotrophins and their receptors in early development of the mammalian nervous system. Acta Neurobiol. Exp. (Wars) 70, 454-467.

Buosi, A.S., Matias, I., Araujo, A.P.B., Batista, C., Gomes, F.C.A., 2017. Heterogeneity in synaptogenic profile of astrocytes from different brain regions. Mol. Neurobiol. 1-12. http://dx.doi.org/10.1007/s12035-016-0343-z.

Cayre, M., Canoll, P., Goldman, J.E., 2009. Cell migration in the normal and pathological postnatal mammalian brain. Prog. Neurobiol. 88, 41-63. http://dx.doi.org/10.1016/ j.pneurobio.2009.02.001.

Cazorla, M., Prémont, J., Mann, A., Girard, N., Kellendonk, C., Rognan, D., 2011. Identification of a low-molecular weight TrkB antagonist with anxiolytic and antidepressant activity in mice. J. Clin. Invest. 121, 1846-1857. http://dx.doi.org/10. $1172 / J C I 43992$.

Choi, S.S., Lee, H.J., Lim, I., Satoh, J., Kim, S.U., 2014. Human astrocytes: Secretome profiles of cytokines and chemokines. PLoS One 9, e92325. http://dx.doi.org/10 1371/journal.pone.0092325.

Cirillo, G., Cavaliere, C., Bianco, M.R., De Simone, A., Colangelo, A.M., Sellitti, S., Alberghina, L., Papa, M., 2010. Intrathecal NGF administration reduces reactive astrocytosis and changes neurotrophin receptors expression pattern in a rat model of neuropathic pain. Cell. Mol. Neurobiol. 30, 51-62. http://dx.doi.org/10.1007/ s10571-009-9430-2.

Colombo, E., Cordiglieri, C., Melli, G., Newcombe, J., Krumbholz, M., Parada, L.F.,
Medico, E., Hohlfeld, R., Meinl, E., Farina, C., 2012. Stimulation of the neurotrophin receptor TrkB on astrocytes drives nitric oxide production and neurodegeneration. J. Exp. Med. 209, 521-535. http://dx.doi.org/10.1084/jem.20110698.

Cragnolini, A.B., Huang, Y., Gokina, P., Friedman, W.J., 2009. Nerve growth factor at tenuates proliferation of astrocytes via the p75 neurotrophin receptor. Glia 57, 1386-1392. http://dx.doi.org/10.1002/glia.20857.

Cragnolini, A.B., Volosin, M., Huang, Y., Friedman, W.J., 2012. Nerve growth factor induces cell cycle arrest of astrocytes. Dev. Neurobiol. 72, 766-776. http://dx.doi.org/ 10.1002/dneu.20981.

Das, K., Chao, S., White, L., Haines, W., Harry, G., Tilson, H., Barone, S., 2001. Differential patterns of nerve growth factor, brain-derived neurotrophic factor and neurotrophin-3 mRNA and protein levels in developing regions of rat brain. Neuroscience 103, 739-761. http://dx.doi.org/10.1016/S0306-4522(01)00011-2.

Dauth, S., Grevesse, T., Pantazopoulos, H., Campbell, P.H., Maoz, B.M., Berretta, S., Parker, K.K., 2016. Extracellular matrix protein expression is brain region dependent. J. Comp. Neurol. 524, 1309-1336. http://dx.doi.org/10.1002/cne.23965.

De Luca, C., Savarese, L., Colangelo, A.M., Bianco, M.R., Cirillo, G., Alberghina, L., Papa, M., 2016. Astrocytes and microglia-mediated immune response in maladaptive plasticity is differently modulated by NGF in the ventral horn of the spinal cord following peripheral nerve injury. Cell. Mol. Neurobiol. 36, 37-46. http://dx.doi.org/ 10.1007/s10571-015-0218-2.

Diniz, D.G., de Oliveira, M.A., de Lima, C.M., Fôro, C.A.R., Sosthenes, M.C.K., BentoTorres, J., da Costa Vasconcelos, P.F., Anthony, D.C., Diniz, C.W.P., 2016. Age, environment, object recognition and morphological diversity of GFAP-immunolabeled astrocytes. Behav. Brain Funct. 12, 28. http://dx.doi.org/10.1186/s12993-0160111-2.

Domeniconi, M., Hempstead, B.L., Chao, M.V., 2007. Pro-NGF secreted by astrocytes promotes motor neuron cell death. Mol. Cell. Neurosci. 34, 271-279. http://dx.doi. org/10.1016/j.mcn.2006.11.005.

Douglas-escobar, M., Rossignol, C., Steindler, D., Zheng, T., Weiss, M.D., 2012. Neurotrophin-induced Migration and Neuronal Differentiation of Multipotent Astrocytic Stem Cells In Vitro. 7. pp. 1-8. http://dx.doi.org/10.1371/journal.pone. 0051706.

Emsley, J.G., Macklis, J.D., 2006. Astroglial heterogeneity closely reflects the neuronaldefined anatomy of the adult murine CNS. Neuron Glia Biol. 2, 175-186. http://dx. doi.org/10.1017/S1740925X06000202.

Etienne-Manneville, S., 2008. Polarity proteins in migration and invasion. Oncogene 27, 6970-6980. http://dx.doi.org/10.1038/onc.2008.347.

Fitting, S., Zou, S., Chen, W., Vo, P., Hauser, K.F., Knapp, P.E., 2010. Regional heterogeneity and diversity in cytokine and chemokine production by astroglia: differential responses to HIV-1 tat, gp120, and morphine revealed by multiplex analysis. J. Proteome Res. 9, 1795-1804. http://dx.doi.org/10.1021/pr900926n.

Friedman, W.J., 2000. Neurotrophins induce death of hippocampal neurons via the p75 receptor. J. Neurosci. 20, 6340-6346.

Fulmer, C.G., VonDran, M.W., Stillman, A.A., Huang, Y., Hempstead, B.L., Dreyfus, C.F. 2014. Astrocyte-derived BDNF supports myelin protein synthesis after Cuprizoneinduced demyelination. J. Neurosci. 34, 8186-8196. http://dx.doi.org/10.1523/ JNEUROSCI.4267-13.2014.

Giralt, A., Coura, R., Girault, JA., 2016. Pyk2 is essential for astrocytes mobility following brain lesion. Glia 64, 620-634. http://dx.doi.org/10.1002/glia.22952.

Gomes, C., Ferreira, R., George, J., Sanches, R., Rodrigues, D.I., Gonçalves, N., Cunha, R.A., 2013. Activation of microglial cells triggers a release of brain-derived neurotrophic factor (BDNF) inducing their proliferation in an adenosine A2A receptordependent manner: A2A receptor blockade prevents BDNF release and proliferation of microglia. J. Neuroinflammation 10, 780. http://dx.doi.org/10.1186/1742-2094$10-16$.

Goss, J.R., O'Malley, M.E., Zou, L., Styren, S.D., Kochanek, P.M., DeKosky, S.T., 1998. Astrocytes are the major source of nerve growth factor upregulation following traumatic brain injury in the rat. Exp. Neurol. 149, 301-309. http://dx.doi.org/10. 1006/exnr.1997.6712.

Grade, S., Weng, Y.C., Snapyan, M., Kriz, J., Malva, J.O., Saghatelyan, A., 2013. Brainderived neurotrophic factor promotes vasculature-associated migration of neuronal precursors toward the ischemic striatum. PLoS One 8. http://dx.doi.org/10.1371/ journal.pone.0055039.

Hartmann, M., Brigadski, T., Erdmann, K.S., Holtmann, B., Sendtner, M., Narz, F., Lessmann, V., 2004. Truncated TrkB receptor-induced outgrowth of dendritic filopodia involves the p75 neurotrophin receptor. J. Cell Sci. 117, 5803-5814. http://dx. doi.org/10.1242/jcs.01511.

Hayakawa, K., Pham, L.-D.D., Arai, K., Lo, E.H., 2014. Reactive astrocytes promote adhesive interactions between brain endothelium and endothelial progenitor cells via HMGB1 and beta-2 integrin signaling. Stem Cell Res. 12, 531-538. http://dx.doi.org/ 10.1016/j.scr.2013.12.008.

Hutton, L.A., DeVellis, J., Perez-Polo, J.R., 1992. Expression of p75NGFR trkA, and trkB mRNA in rat C6 glioma and type I astrocyte cultures. J. Neurosci. Res. 32, 375-383. http://dx.doi.org/10.1002/jnr.490320309.

Islam, O., Loo, T.X., Heese, K., 2009. Brain-derived neurotrophic factor (BDNF) has proliferative effects on neural stem cells through the truncated TRK-B receptor, MAP kinase, AKT, and STAT-3 signaling pathways. Curr. Neurovasc. Res. 6, 42-53. http:// dx.doi.org/10.2174/156720209787466028.

Johnson, K.M., Milner, R., Crocker, S.J., 2015. Extracellular matrix composition determines astrocyte responses to mechanical and inflammatory stimuli. Neurosci. Lett. 600, 104-109. http://dx.doi.org/10.1016/j.neulet.2015.06.013.

Kawano, H., Kimura-Kuroda, J., Komuta, Y., Yoshioka, N., Li, H.P., Kawamura, K., Li, Y., Raisman, G., 2012. Role of the lesion scar in the response to damage and repair of the central nervous system. Cell Tissue Res. 349, 169-180. http://dx.doi.org/10.1007/ s00441-012-1336-5. 
Környei, Z., Czirók, A., Vicsek, T., Madarász, E., 2000. Proliferative and migratory responses of astrocytes to in vitro injury. J. Neurosci. Res. 61, 421-429. http://dx.doi. org/10.1002/1097-4547(20000815)61:4<421::AID-JNR8> 3.0.CO;2-4.

Lu, J., Frerich, J.M., Turtzo, L.C., Li, S., Chiang, J., Yang, C., Wang, X., Zhang, C., Wu, C., Sun, Z., Niu, G., Zhuang, Z., Brady, R.O., Chen, X., 2013. Histone deacetylase inhibitors are neuroprotective and preserve NGF-mediated cell survival following traumatic brain injury. Proc. Natl. Acad. Sci. U. S. A. 110, 10747-10752. http://dx. doi.org/10.1073/pnas.1308950110.

Matyas, J.J., O'Driscoll, C.M., Yu, L., Coll-Miro, M., Daugherty, S., Renn, C.L., Faden, A.I., Dorsey, S.G., Wu, J., 2017. Truncated TrkB.T1-mediated astrocyte dysfunction contributes to impaired motor function and neuropathic pain after spinal cord injury. J. Neurosci. 37, 3353-3971. http://dx.doi.org/10.1523/JNEUROSCI.3353-16.2017.

McCarthy, K., De Vellis, J., 1980. Preparation of seperate astroglial and oligodendroglial cultures from rat cerebral tissue. J. Cell Biol. 85, 890-902 (doi:0021-9525/80).

Oderfeld-Nowak, B., Orzyłowska-Śliwińska, O., Sołtys, Z., Zaremba, M., Januszewski, S., Janeczko, K., Mossakowski, M., 2003. Concomitant up-regulation of astroglial high and low affinity nerve growth factor receptors in the CA1 hippocampal area following global transient cerebral ischemia in rat. Neuroscience 120, 31-40. http://dx.doi. org/10.1016/S0306-4522(03)00289-6.

Ohira, K., Funatsu, N., Homma, K.J., Sahara, Y., Hayashi, M., Kaneko, T., Nakamura, S., 2007. Truncated TrkB-T1 regulates the morphology of neocortical layer I astrocytes in adult rat brain slices. Eur. J. Neurosci. 25, 406-416. http://dx.doi.org/10.1111/j. 1460-9568.2007.05282.x.

Oliveira, S.L.B., Pillat, M.M., Cheffer, A., Lameu, C., Schwindt, T.T., Ulrich, H., 2013 Functions of neurotrophins and growth factors in neurogenesis and brain repair. Cytometry A 83 A, 76-89. http://dx.doi.org/10.1002/cyto.a.22161.

Park, H., Poo, M., 2012. Neurotrophin regulation of neural circuit development and function. Nat. Rev. Neurosci. 14, 7-23. http://dx.doi.org/10.1038/nrn3379.

Patel, A.J., Gray, C.W., 1993. Neurotrophic factors produced by astrocytes involved in the regulation of cholinergic neurons in the central nervous system. In: Biology and Pathology of Astrocyte-Neuron Interactions. Springer US, Boston, MA, pp. 103-115. http://dx.doi.org/10.1007/978-1-4757-9486-1_9.

Pekny, M., Pekna, M., 2014. Astrocyte reactivity and reactive astrogliosis: costs and benefits. Physiol. Rev. 94, 1077-1098. http://dx.doi.org/10.1152/physrev.00041. 2013.

Rubio, N., 1997. Mouse astrocytes store and deliver brain-derived neurotrophic factor using the non-catalytic gp95 ${ }^{\text {trkB }}$ receptor. Eur. J. Neurosci. 9, 1847-1853. http://dx. doi.org/10.1111/j.1460-9568.1997.tb00751.x.

Rudge, J.S., Li, Y., Pasnikowski, E.M., Mattsson, K., Pan, L., Yancopoulos, G.D., Wiegand, S.J., Lindsay, R.M., Ip, N.Y., 1994. Neurotrophic factor receptors and their signa transduction capabilities in rat astrocytes. Eur. J. Neurosci. 6, 693-705.

Schachtrup, C., Ryu, J.K., Mammadzada, K., Khan, A.S., Carlton, P.M., Perez, A., Christian, F., Le Moan, N., Vagena, E., Baeza-Raja, B., Rafalski, V., Chan, J.P., Nitschke, R., Houslay, M.D., Ellisman, M.H., Wyss-Coray, T., Palop, J.J., Akassoglou, K., 2015. Nuclear pore complex remodeling by p75NTR cleavage controls TGF- $\beta$ signaling and astrocyte functions. Nat. Neurosci. 18, 1077-1080. http://dx.doi.org/ 10.1038/nn.4054.

Schindelin, J., Arganda-Carreras, I., Frise, E., Kaynig, V., Longair, M., Pietzsch, T. Preibisch, S., Rueden, C., Saalfeld, S., Schmid, B., Tinevez, J.-Y., White, D.J., Hartenstein, V., Eliceiri, K., Tomancak, P., Cardona, A., 2012. Fiji: an open-source platform for biological-image analysis. Nat. Methods 9, 676-682. http://dx.doi.org/ 10.1038/nmeth.2019.

Schitine, C., Nogaroli, L., Costa, M.R., Hedin-Pereira, C., 2015. Astrocyte heterogeneity in the brain: from development to disease. Front. Cell. Neurosci. 9, 76. http://dx.doi. org/10.3389/fncel.2015.00076.

Shonukan, O., Bagayogo, I., McCrea, P., Chao, M., Hempstead, B., 2003. Neurotrophininduced melanoma cell migration is mediated through the actin-bundling protein fascin. Oncogene 22, 3616-3623. http://dx.doi.org/10.1038/sj.onc.1206561.

Sofroniew, M.V., 2015. Astrogliosis. Cold Spring Harb. Perspect. Biol. 7, a020420. http:// dx.doi.org/10.1101/cshperspect.a020420.

Tay, T.L., Savage, J., Hui, C.W., Bisht, K., Tremblay, M.-È., 2016. Microglia across the lifespan: from origin to function in brain development, plasticity and cognition. J. Physiol. 0, 1-44. http://dx. doi.org/10.1113/JP272134.

Tu, Z., Li, Y., Dai, Y., Li, L., Lv, G., Chen, I., Wang, B., 2017. MiR-140/BDNF axis regulates normal human astrocyte proliferation and LPS-induced IL- 6 and TNF- $\alpha$ secretion. Biomed Pharmacother 91, 899-905. http://dx.doi.org/10.1016/j.biopha.2017.05. 016.

Volosin, M., Song, W., Almeida, R.D., Kaplan, D.R., Hempstead, B.L., Friedman, W.J., 2006. Interaction of survival and death signaling in basal forebrain neurons: roles of neurotrophins and proneurotrophins. J. Neurosci. 26, 7756-7766. http://dx.doi.org/ 10.1523/JNEUROSCI.1560-06.2006.

VonDran, M.W., LaFrancois, J., Padow, V.A., Friedman, W.J., Scharfman, H.E., Milner, T.A., Hempstead, B.L., 2014. p75NTR, but not proNGF, is upregulated following status epilepticus in mice. ASN Neuro 6. http://dx.doi.org/10.1177/ 1759091414552185.

Waterhouse, E.G., An, J.J., Orefice, L.L., Baydyuk, M., Liao, G.-Y., Zheng, K., Lu, B., Xu, B., 2012. BDNF promotes differentiation and maturation of adult-born neurons through GABAergic transmission. J. Neurosci. 32, 14318-14330. http://dx.doi.org/ 10.1523/JNEUROSCI.0709-12.2012.

Wiese, S., Karus, M., Faissner, A., 2012. Astrocytes as a source for extracellular matrix molecules and cytokines. Front. Pharmacol. 3, 120. http://dx.doi.org/10.3389/fphar. 2012.00120.

Wu, J., Renn, C.L., Faden, A.I., Dorsey, S.G., 2013. TrkB.T1 contributes to neuropathic pain after spinal cord injury through regulation of cell cycle pathways. J. Neurosci. 33, 12447-12463. http://dx.doi.org/10.1523/JNEUROSCI.0846-13.2013.

Yang, H., Cheng, X.-P., Li, J.-W., Yao, Q., Ju, G., 2009. De-differentiation response of cultured astrocytes to injury induced by scratch or conditioned culture medium of scratch-insulted astrocytes. Cell. Mol. Neurobiol. 29, 455-473. http://dx.doi.org/10 1007/s10571-008-9337-3.

Zhan, J.S., Gao, K., Chai, R.C., Jia, X.H., Luo, D.P., Ge, G., Jiang, Y.W., Fung, Y. wan W., Li, L., Yu, A.C.H., 2017. Astrocytes in migration. Neurochem. Res. 42, 272-282. http://dx.doi.org/10.1007/s11064-016-2089-4. 